\title{
HIGHER MONOTONICITY PROPERTIES AND INEQUALITIES FOR ZEROS OF BESSEL FUNCTIONS
}

\author{
LAURA NICOLO-AMATI GORI, ANDREA LAFORGIA, \\ AND MARTIN E. MULDOON
}

(Communicated by Kenneth R. Meyer)

\begin{abstract}
L. Lorch and P. Szegö have considered the sign-regularity of the higher differences (with respect to the rank $k$ ) of the sequence $\left\{c_{\nu k}\right\}$ of positive zeros of the Bessel function $\mathscr{C}_{\nu}(x)$. Our main purpose here is to extend one of their main results to the higher derivatives with respect to $\kappa$ when $c_{\nu k}$ is appropriately defined as a function of a continuous variable $\kappa$ rather than the discrete variable $k$, and the difference operator is replaced by a derivative operator. We also present some inequalities arising from these and other results.
\end{abstract}

\section{INTRODUCTION}

L. Lorch and P. Szegö [6] initiated the study of the sign-regularity of higher differences with respect to the rank $k$, of the sequence $\left\{c_{\nu k}\right\}=\left\{c_{\nu k}(\alpha)\right\}$ of positive zeros of the cylinder function

$$
\mathscr{C}_{\nu}(x)=\mathscr{C}_{\nu}(x, \alpha)=\cos \alpha J_{\nu}(x)-\sin \alpha Y_{\nu}(x), \quad 0 \leq \alpha<\pi .
$$

In particular they showed that for $|\nu|>1 / 2$, we have

$$
(-1)^{n} \Delta^{n+1} c_{\nu k}>0, \quad n=0,1, \ldots, k=1,2, \ldots
$$

This result was generalized and extended in [7], [8] and several papers by other authors. The main purpose of this note is to show that the result remains true when $c_{\nu k}$ is appropriately defined as a function of a continuous variable $\kappa$ rather than the discrete variable $k$, and the difference operator is replaced by a derivative operator. We also present several other monotonicity properties and inequalities for $c_{\nu k}$.

\section{Defining the zeros as Continuous functions of $k$}

One can discuss the variation of the positive zeros of $\mathscr{C}_{\nu}(x, \alpha)$ with respect to any of the three variables $\nu, \alpha$ or $k$ (the rank of a zero). However, $\alpha$

Received by the editors November 30, 1989 and, in revised form, July 31, 1990.

1980 Mathematics Subject Classification (1985 Revision). Primary 33A40.

Key words and phrases. Bessel functions, zeros, higher monotonicity, inequalities.

The authors' work was supported by grants from Consiglio Nazionale delle Ricerche (Italy) and by the Natural Sciences and Engineering Research Council (Canada). 
and $k$ are not really independent; they may, in fact, be subsumed in a single variable $\kappa=k-\alpha / \pi$. To see this, we consider that for $\nu \geq 0$, the zeros of $\mathscr{C}_{\nu}(x, \alpha), 0<\alpha<\pi$, are the roots of the equation

$$
Y_{\nu}(x) / J_{\nu}(x)=\cot \alpha \text {. }
$$

The graph of the left-hand side of (2.1) consists of branches which increase from $-\infty$ to $+\infty$ in the intervals $\left(0, j_{\nu 1}\right)$ and $\left(j_{\nu k}, j_{\nu, k+1}\right), k=1,2, \ldots$, between the positive zeros of $J_{\nu}(x)$. This is most easily seen by using the relation

$$
\frac{d}{d x} \frac{Y_{\nu}(x)}{J_{\nu}(x)}=\frac{J_{\nu}(x) Y_{\nu}^{\prime}(x)-Y_{\nu}(x) J_{\nu}^{\prime}(x)}{J_{\nu}^{2}(x)}=\frac{2}{\pi x J_{\nu}^{2}(x)},
$$

where the last equation follows from the Wronskian relation [11, p. 76]. As $\alpha$ decreases from $\pi$ to $0, \cot \alpha$ increases from $-\infty$ to $\infty$. Thus each zero of $\mathscr{C}_{\nu}(x, \alpha)$ increases from one positive zero $j_{\nu k}$ of $J_{\nu}(x)$ to the next larger one $j_{\nu, k+1}$. At the same time a new first positive zero appears and increases from 0 to $j_{\nu 1}$. Thus it makes sense to define $j_{\nu \kappa}$ for any real $\kappa \geq 0$, by $j_{\nu 0}=0$ and $j_{\nu \kappa}=c_{\nu k}(\alpha)$ where $k$ is the largest integer less than $\kappa+1$ and $\alpha=\pi(k-\kappa)$. Thus $j_{\nu \kappa}$ is a continuous increasing function of $\kappa$ on $[0, \infty)$. The positive zeros of $J_{\nu}(x)$ correspond to positive integral values of $\kappa$ and $j_{\nu, k-1 / 2}=y_{\nu k}, k=1,2, \ldots$ where $y_{\nu k}$ is the $k$ th positive zero of $Y_{\nu}(x)$. In [2] it was shown that $j_{\nu \kappa}$ is the unique solution of the differential equation

$$
\frac{d j}{d \nu}=2 j \int_{0}^{\infty} K_{0}(2 j \sinh t) e^{-2 \nu t} d t
$$

which satisfies $j(\nu) \rightarrow 0$ as $\nu \rightarrow-\kappa^{+}$. This is motivated by the formula [11, p. 408] for the derivative of $c_{\nu k}$ with respect to $\nu$, and the fact that if, for $\nu>0, c_{\nu k}$ is the $k$ th positive zero of $\mathscr{C}_{\nu}(x)$, then $c_{\nu k}$ may be extended in a continuous way to $\nu<0$, and $c_{\nu k} \rightarrow 0$, as $\nu \rightarrow-(k-\alpha / \pi)$. The equation (2.2) may be used to show that $j_{\nu \kappa}$ is an infinitely differentiable function of $\kappa$.

We shall often use the fact that $j_{1 / 2, \kappa}=\kappa \pi$.

\section{THE CASE $\nu>1 / 2$; COMPLETE MONOTONICITY}

Here we show

Theorem 3.1. Let $j_{\nu \kappa}$ be defined as in $\S 2$. Then, for $\nu>\mu \geq 1 / 2$,

$$
(-1)^{n} D_{\kappa}^{n}\left(\log \left[j_{\nu \kappa} / j_{\mu \kappa}\right]\right)>0, \quad \kappa>0, n=0,1, \ldots,
$$

and, in particular, for $\nu>1 / 2$,

$$
(-1)^{n} D_{\kappa}^{n}\left(\log \left[j_{\nu \kappa} /(\kappa \pi)\right]\right)>0, \quad \kappa>0, n=0,1, \ldots
$$

Theorem 3.2. Let $j_{\nu \kappa}$ be defined as in $\S 2$. Then, for $\nu>\mu \geq 1 / 2$,

$$
(-1)^{n} D_{\kappa}^{n}\left(\log \left[D_{\kappa} j_{\nu \kappa} / D_{\kappa} j_{\mu \kappa}\right]\right)>0, \quad \kappa>0, n=0,1, \ldots,
$$

and, in particular, for $\nu>1 / 2$,

$$
(-1)^{n} D_{\kappa}^{n}\left(\log \left[D_{\kappa} j_{\nu \kappa} / \pi\right]\right)>0, \quad \kappa>0, n=0,1, \ldots
$$


Corollary 3.3. Let $j_{\nu \kappa}$ be defined as in $\S 2$. Then, for $\nu>1 / 2$,

$$
(-1)^{n} D_{\kappa}^{n+1}\left(j_{\nu \kappa}\right)>0, \quad \kappa>0, n=0,1, \ldots
$$

Proofs. We have, from (2.2),

$$
\frac{1}{j_{\lambda \kappa}} \frac{d j_{\lambda \kappa}}{d \lambda}=2 \int_{0}^{\infty} K_{0}\left(2 j_{\lambda \kappa} \sinh t\right) e^{-2 \lambda t} d t
$$

Hence, integrating with respect to $\lambda$ from $\mu$ to $\nu$, we get

$$
\log \frac{j_{\nu \kappa}}{j_{\mu \kappa}}=2 \int_{\mu}^{\nu}\left\{\int_{0}^{\infty} K_{0}\left(2 j_{\lambda \kappa} \sinh t\right) e^{-2 \lambda t} d t\right\} d \lambda .
$$

Next we use the result [3, p. 1484] of Elbert and Laforgia that

$$
D_{\nu} \log D_{\kappa} j_{\nu \kappa}=2 \int_{0}^{\infty} K_{0}\left(2 j_{\nu \kappa} \sinh t\right)\left(\tanh ^{2} t+2 \nu \tanh t\right) e^{-2 \nu t} d t
$$

whence it follows that

(3.8) $\log \left[D_{\kappa} j_{\nu \kappa} / D_{\kappa} j_{\mu \kappa}\right]$

$$
=2 \int_{\mu}^{\nu}\left\{\int_{0}^{\infty} K_{0}\left(2 j_{\lambda \kappa} \sinh t\right)\left(\tanh ^{2} t+2 \lambda \tanh t\right) e^{-2 \lambda t} d t\right\} d \lambda,
$$

and, in particular, when $\mu=1 / 2$,

$$
\begin{aligned}
& \log \left[D_{\kappa} j_{\nu \kappa}\right] \\
& \quad=\log \pi+2 \int_{1 / 2}^{\nu}\left\{\int_{0}^{\infty} K_{0}\left(2 j_{\lambda \kappa} \sinh t\right)\left(\tanh ^{2} t+2 \lambda \tanh t\right) e^{-2 \lambda t} d t\right\} d \lambda .
\end{aligned}
$$

Proving Theorems 3.1 and 3.2 amounts to showing that

$$
\log \left[j_{\nu \kappa} / j_{\mu \kappa}\right] \in \mathscr{M}_{n}(0, \infty),
$$

and

$$
\log \left[D_{\kappa} j_{\nu \kappa} / D_{\kappa} j_{\mu \kappa}\right] \in \mathscr{M}_{n}(0, \infty),
$$

for $n=1,2, \ldots$, where $\mathscr{M}_{n}(0, \infty)$ is the set of functions $f$ on $(0, \infty)$ satisfying

$$
(-1)^{j} f^{(j)}(\kappa)>0, \quad j=0, \ldots, n .
$$

Recall that

$$
(-1)^{n} D_{t}^{n} K_{0}(t)>0, \quad t>0, n=0,1, \ldots
$$

This follows from the integral representation [11, p. 172]

$$
K_{0}(x)=\int_{0}^{\infty} e^{-x \cosh t} d t .
$$

We will in fact show that, for each $a>0$, and $\nu \geq 1 / 2$,

$$
K_{0}\left(a j_{\nu \kappa}\right) \in \mathscr{M}_{n}(0, \infty) \text {, }
$$


for $n=0,1, \ldots$, and the representations (3.6), (3.8) will then give Theorems 3.1 and 3.2. Now in the case $\nu=1 / 2,(3.13)$ follows, for $n=0,1, \ldots$ from (3.12). We prove (3.13) by induction in the case $\nu>1 / 2$. In this case (3.13) is obvious for $n=0,1$; this follows from (3.12) and the fact that $j_{\nu \kappa}$ increases as $\kappa$ increases. Suppose that (3.13) holds for $n=N-1$. Then, from (3.9), we see that, for $\nu>1 / 2$,

$$
\log D_{\kappa} j_{\nu \kappa} \in \mathscr{M}_{N-1}(0, \infty)
$$

Since the exponential of a function in $\mathscr{M}_{n}(0, \infty)$ is also in $\mathscr{M}_{n}(0, \infty)$, we have

$$
(-1)^{n} D_{\kappa}^{n+1}\left(j_{\nu \kappa}\right)>0, \quad \kappa>0, n=0, \ldots, N-1 .
$$

By the formula of Faa di Bruno (see, e.g., [5, $§ 81$, pp. 92-93]) for the $N$ th derivative of a function of a function, we have, for each $a>0$,

$$
(-1)^{N} D_{\kappa}^{N}\left\{K_{0}\left(a j_{\nu \kappa}\right)\right\}>0 \text {. }
$$

To see this we use (3.12) and (3.15) to show that each term arising from the Faa di Bruno formula is nonnegative and at least one is positive. See [7, Proof of Lemma 2.1] for details. Now (3.16) shows that (3.13) holds for $n=N$. Thus the proof by induction is complete and (3.13) holds for $n=0,1, \ldots$. The representations (3.6), (3.8) now give Theorems 3.1 and 3.2.

Corollary 3.3 is a consequence of the fact that $(3.15)$ holds for all $N$. It recovers and generalizes the result of $[3, \mathrm{p} .1485]$ that $j_{\nu \kappa}$ is concave with respect to $\kappa$ when $\nu \geq 1 / 2$. Corollary 3.3 is the continuous version of the result of Lorch and Szegö [6] referred to in the Introduction. That result may be deduced from Corollary 3.3 by using a mean-value theorem for higher derivatives and differences ([10, no. 98] or [5, p. 74]).

The results of this section provide some examples of infinitely divisible distributions. Recall that a probability distribution is infinitely divisible if and only if its Laplace transform is of the form $e^{-h(x)}$, where $h(0)=0$ and $h^{\prime}(x)$ is completely monotonic [4, p. 425]. Thus, from Theorem 3.1, we can deduce the complete monotonicity of the distribution whose Laplace transform is $j_{\mu, s+\epsilon} j_{\nu, \epsilon} / j_{\nu, s+\epsilon} j_{\mu, \epsilon}$, where $\nu>\mu \geq 1 / 2, \epsilon>0$ and $s$ is the variable in the Laplace transform.

\section{The CASE $0 \leq \nu<1 / 2$; SIMPLE MONOTONICITY}

It does not seem to be easy to derive higher monotonicity results in the case when $0 \leq \nu<1 / 2$. In [6] it was conjectured that (1.1) should be replaced by

$$
(-1)^{n} \Delta^{n+2} c_{\nu k}>0, \quad n=0,1, \ldots, k=1,2, \ldots
$$

in the case $|\nu|<1 / 2$. In [9], some progress was made in this direction when it was shown that (4.1) holds for $1 / 3 \leq|\nu|<1 / 2$. There does not seem to be any easy way to get the analogues of the results of $\S 3$ in the case $0 \leq \nu<1 / 2$. The condition $\nu>1 / 2$ is an essential ingredient in getting (3.14), for example. However, we are able to deduce some simple monotonicity results in this case. 
Theorem 4.1. Let $j_{\nu \kappa}$ be defined as in $\S 2$. Then, if $0 \leq \mu<\nu \leq 1 / 2$, the positive function $j_{\nu \kappa} / j_{\mu \kappa}$ decreases to 1 as $\kappa$ increases on $(0, \infty)$. If $0 \leq \mu<1 / 2$, then $j_{\mu \kappa} /(\kappa \pi)$ increases with $\kappa, 0<\kappa<\infty$.

Theorem 4.2. Let $j_{\nu \kappa}$ be defined as in $\S 2$. Then, if $0 \leq \mu<\nu \leq 1 / 2$, the positive function $D_{\kappa} j_{\nu \kappa} / D_{\kappa} j_{\mu \kappa}$ decreases to 1 as $\kappa$ increases on $(0, \infty)$. If $0 \leq \mu<1 / 2$, then $D_{\kappa} j_{\mu \kappa}$ increases with $\kappa, 0<\kappa<\infty$.

Remark. It should be noted that the monotonicities recorded here in the case $\mu<1 / 2$ are opposite in direction to those which we get, in the case of order $>1 / 2$, as a consequence of the theorems in $\S 3$.

The main assertions of Theorems 4.1 and 4.2 follow easily from the representations (3.6) and (3.8) once we recall that $K_{0}$ is a decreasing function of its argument and that $j_{\nu \kappa}$ increases as $\kappa$ increases. The final assertions of the theorems follow on putting $\nu=1 / 2$.

\section{Derivative OF A ZERO With RESPECT TO $\kappa$}

We obviously have $d j_{\nu \kappa} / d \nu>0$. Here we show

Theorem 5.1.

$$
\begin{array}{cc}
d j_{\nu \kappa} / d \kappa>\pi, & \nu>1 / 2, \\
d j_{\nu \kappa} / d \kappa<\pi, & 0 \leq \nu<1 / 2 .
\end{array}
$$

Proof. From the MacMahon expansion [11, p. 506],

$$
j_{\nu \kappa}=(\kappa+\nu / 2-1 / 4) \pi+O\left(\kappa^{-1}\right), \quad \kappa \rightarrow \infty,
$$

we have $j_{\nu \kappa} / \kappa \rightarrow \pi$, as $\kappa \rightarrow \infty$. Hence, by an indirect application of L'Hospital's rule, we see that $d j_{\nu \kappa} / d \kappa \rightarrow \pi$, as $\kappa \rightarrow \infty$. Now, for $\nu>1 / 2$, $j_{\nu \kappa}$ is a concave function of $\kappa$, by Corollary 3.3. Hence, $d j_{\nu \kappa} / d \kappa$ decreases and (5.1) follows. On the other hand, when $0 \leq \nu<1 / 2, d j_{\nu \kappa} / d \kappa$ increases, by Theorem 4.2, and (5.2) follows.

Remark. The same indirect use of L'Hospital's rule shows the known result [1, Corollary 3.4, p. 276], that $d j_{\nu \kappa} / d \nu>1$, where $\kappa$ is so large that $j_{\nu \kappa}$ is a concave function of $\nu$.

The principal term of (5.3) gives an upper bound for $j_{\nu \kappa}$ in case $\nu>1 / 2$ and a lower bound in case $0 \leq \nu<1 / 2$. To see this we write

$$
f(\kappa)=j_{\nu \kappa}-(\kappa+\nu / 2-1 / 4) \pi
$$

and note from [2, Theorem 2.1], or from Theorem 5.1 above, that $f(\kappa)$ is increasing, constant or decreasing according as $\nu>1 / 2, \nu=1 / 2$ or $0 \leq \nu<$ $1 / 2$. Also $f(\kappa) \rightarrow 0$, as $\kappa \rightarrow \infty$ by (5.3), so the stated results follow.

We establish now some consequences of Theorem 5.1. Integration of (5.1) between $\kappa_{0}$ and $\kappa, \kappa>\kappa_{0}>0$, gives

$$
j_{\nu \kappa}-j_{\nu \kappa_{0}}>\pi\left(\kappa-\kappa_{0}\right), \quad \nu>1 / 2
$$


where the inequality must be reversed when $0 \leq \nu<1 / 2$ and becomes equality when $\nu=1 / 2$. If $j_{\nu \kappa_{0}}$ and $j_{\nu \kappa}$ are two consecutive zeros of the same cylinder function, we find the known result that the distance between two consecutive zeros is larger than, equal to or less than $\pi$ according as $\nu>1 / 2, \nu=1 / 2$ or $\nu<1 / 2$.

An interesting particular case of (5.4) is when $\kappa_{0}=n-1 / 2$ and $\kappa=n$. In this case we find that

$$
j_{\nu, n}>y_{\nu, n}+\pi / 2, \quad \nu>1 / 2, n=1,2, \ldots
$$

Clearly other inequalities of this kind can be found taking into consideration, in (5.4), different particular values of $\kappa_{0}$ and $\kappa$.

\section{INEQUALITIES ARISING FROM CONVEXITY OR CONCAVITY WITH RESPECT TO $\kappa$}

The function $j_{\nu \kappa}$ is known to be concave (convex) with respect to $\kappa, 0<$ $\kappa<\infty$, for $\nu>1 / 2(0 \leq \nu<1 / 2)$ [3, p. 1485]. By considering the chord joining two points on the graph of this function, we thus obtain:

$$
j_{\nu \kappa}>\frac{\kappa-k}{K-k}\left(j_{\nu K}-j_{\nu k}\right)+j_{\nu k}, \quad k<\kappa<K, \nu>1 / 2,
$$

and

$$
j_{\nu \kappa}<\frac{\kappa-k}{K-k}\left(j_{\nu K}-j_{\nu k}\right)+j_{\nu k}, \quad k<\kappa<K, 0 \leq \nu<1 / 2 .
$$

These become equalities when $\nu=1 / 2$.

If we put $k=n, K=n+1$, we get

$$
\begin{array}{cc}
j_{\nu \kappa}>(\kappa-n)\left(j_{\nu, n+1}-j_{\nu n}\right)+j_{\nu n}, & \nu>1 / 2, \\
j_{\nu \kappa}<(\kappa-n)\left(j_{\nu, n+1}-j_{\nu n}\right)+j_{\nu n}, & 0 \leq \nu<1 / 2,
\end{array}
$$

In the case where $\kappa=n+1 / 2$, we get

$$
\begin{array}{cc}
y_{\nu, n+1}>\left(j_{\nu, n+1}+j_{\nu n}\right) / 2, & \nu>1 / 2, \\
y_{\nu, n+1}<\left(j_{\nu, n+1}+j_{\nu n}\right) / 2, & 0 \leq \nu<1 / 2 .
\end{array}
$$

For example, taking $\nu=0, n=39$ in (6.6) gives $y_{0,40}<123.3085255$, where the values of $j_{0,39}, j_{0,40}$ are taken from [11, p. 748]. The "exact" value of $y_{0,40}$, from the same source, is 123.3085253 .

On the other hand, if in (6.2) we take $k=n-1 / 2, \kappa=n, K=n+1 / 2$, we get

$$
j_{\nu, n}<\left(y_{\nu, n+1}+y_{\nu n}\right) / 2, \quad \nu<1 / 2 .
$$

In particular, with $n=39, \nu=0$, we get $y_{0,40}>2 j_{0,39}-y_{0,39}=123.308525$. 
The upper and lower bounds

$$
123.308525<y_{0,40}<123.3085255
$$

show the sharpness of these inequalities.

We have found the inequalities to be similarly sharp in the case $\nu=1$ and even more so in the case $\nu=1 / 3$, as may be expected from the fact that they become equalities when $\nu=1 / 2$.

Inequalities of a similar kind may be obtained by using, for example, the increase of $j_{\nu \kappa} / \kappa$ with respect to $\kappa(0<\kappa<\infty)$ for $0 \leq \nu<1 / 2$ (Theorem 4.1). Thus, for example, with $\nu=1 / 3$, we get

or

$$
\frac{y_{1 / 3,40}}{39.5}=\frac{j_{1 / 3,39.5}}{39.5}>\frac{j_{1 / 3,39}}{39}
$$

$$
y_{1 / 3,40}>(79 / 78) j_{1 / 3,39}=123.8283293 \text {; }
$$

the "actual" value of $y_{1 / 3,40}$ is 123.8316712 . The numerical values are from [11, p. 75].

An advantage of the results enunciated here is that the knowledge of the zeros of one cylinder function ( $J_{\nu}(x)$, for example) enables us to establish approximations for the zeros of any other cylinder function, those of $Y_{\nu}(x)$ being but one example. Also, some of the inequalities found in this section are more informative than some of those which can be obtained by a direct application of the Sturm comparison theorem. For example, when $\nu>1 / 2$, one can use the Sturm theorem to show that $y_{\nu, n+1}$ belongs to the interval $\left(j_{\nu, n}, j_{\nu, n+1}\right)$, but formula (6.5) asserts that it belongs to the second half of this interval.

\section{ACKNOWLEDGMENT}

We are grateful to a referee for several constructive comments.

\section{REFERENCES}

1. A. Elbert, L. Gatteschi, and A. Laforgia, On the concavity of zeros of Bessel functions, Appl. Anal. 16 (1983), 261-278.

2. A. Elbert and A. Laforgia, On the square of the zeros of Bessel functions, SIAM J. Math. Anal. 15 (1984), 206-212.

3. (1986), 1483-1488.

4. W. Feller, An introduction to probability theory and its applications, vol. 2, Wiley, 1966.

5. C. J. de La Vallée Poussin, Cours d'analyse infinitésimale, 12th ed., Louvain and Paris, 1959.

6. L. Lorch and P. Szegö, Higher monotonicity properties of certain Sturm-Liouville functions, Acta Math. 109 (1963), 55-73.

7. L. Lorch, M. E. Muldoon, and P. Szegö, Higher monotonicity properties of certain SturmLiouville functions. III, Canad. J. Math. 22 (1970), 1238-1265.

8. _ Higher monotonicity properties of certain Sturm-Liouville functions. IV, Canad. J. Math. 24 (1972), 349-368. 
9. M. E. Muldoon, Higher monotonicity properties of certain Sturm-Liouville functions. V, Proc. Roy. Soc. Edinburgh Sect. A 77 (1977), 23-37.

10. G. Pólya and G. Szegö, Problems and theorems in analysis II, Springer-Verlag, 1972.

11. G. N. Watson, A treatise on the theory of Bessel functions, 2nd ed., Cambridge University Press, 1944.

Dipartimento di Metodi e Modelli Matematici per le Scienze Applicate, Universita DEgli Studi di Roma "LA SAPIENZA", Via A. Scarpa, 10, I-00161 Roma, Italy

Dipartimento di Matematica ed Applicazioni, Universita di Palermo, Via Archirafi, 34, I-90123 Palermo, Italy

Current address: Facoltá d'Ingegneria dell'Universitá dell'Aquila, Monteluco di Roio, I-67040 L'Aquila, Italy

Department of Mathematics, York University, North York, Ontario M3J 1P3, Canada 